\title{
Using NLP for Enhancing Second Language Acquisition
}

Leonardo Zilio

\author{
CENTAL \\ Université catholique de Louvain \\ \{leonardo.zilio, rodrigo.wilkens, cedrick.fairon\}@uclouvain.be
}

Cédrick Fairon

Rodrigo Wilkens

\begin{abstract}
This study presents SMILLE, a system that draws on the Noticing Hypothesis and on input enhancements, addressing the lack of salience of grammatical information in online documents chosen by a given user. By means of input enhancements, the system can draw the user's attention to grammar, which could possibly lead to a higher intake per input ratio for metalinguistic information. The system receives as input an online document and submits it to a combined processing of parser and hand-written rules for detecting its grammatical structures. The input text can be freely chosen by the user, providing a more engaging experience and reflecting the user's interests. The system can enhance a total of 107 fine-grained types of grammatical structures that are based on the CEFR. An evaluation of some of those structures resulted in an overall precision of $87 \%$.
\end{abstract}

\section{Introduction}

Research on the field of second language acquisition (SLA) has already shown that the mere presentation of input to a language learner is not enough for ensuring that some linguistic information will be retained (Meurers et al., 2010). This means that the language learner may process the input for its meaning alone, without noticing its linguistic structures, because there is no salient grammatical information. Input is, therefore, understood as "potentially processible language data which are made available, by chance or by design, to the language learner" (Smith, 1993). On the other hand, the intake is the part of the input which is actually internalized by the user and that can potentially be connected to the long-term memory (Reinders, 2012).

As such, an input in its raw form has lower chances of being converted into intake by the learner, and may thus not provide any new linguistic information. In the early 90's, Schmidt (1990) developed the hypothesis that, in order to convert input into intake, a language learner needs to notice the relevant information in the input. More recently, Schmidt (2012) stated, in a less controversial way, that "people learn about the things that they attend to and do not learn much about the things they do not attend to". There is much discussion regarding the assumptions of the Noticing Hypothesis, and it has some fierce contesters, such as Truscott (1998). Nevertheless, it seems to be of general agreement that noticing is at least a facilitator of the language learning process, even though there is differences in the way that authors view the process of noticing, either as a purely conscious process or as a possibly unconscious process (Cross, 2002).

To solve the lack of salience in raw input, Smith and Truscott (2014) suggested the use of "input enhancements", so as to give prominence to the relevant linguistic information. This focus-onform strategy (Doughty, 1991) provides a way to assist language learners, and recent studies on SLA have shown that input enhancements represent a positive step in transforming input into intake (Plonsky and Ziegler, 2016; Simard, 2009).

CALL systems that are able to deal with authentic texts and uses NLP for rendering a better presentation of linguistic information are called Authentic Texts Intelligent Computer-Assisted Language Learning (ATICALL) Meurers (2012). In this paper, we present the Smart and Intelligent Language Learning Environment (SMILLE), an ATICALL system that enhances authentic Web pages by using available NLP tools. It extracts the 
text content of a Web page chosen by the user (i.e., the language learner) and processes it, retrieving linguistic information that can be enhanced according to the user's specific language learning needs. To ensure that the highlighted information is relevant, SMILLE is linked to the users' language level, as described by the Common European Framework of Reference for Languages (CEFR) (Council of Europe, 2011).

SMILLE was developed based on a scenario in which the users are already pursuing a foreign language course and wish to continue the language learning activity by means of reading Web-based material that corresponds to their interests. In this case, SMILLE can help not only with the textunderstanding process, for it has built-in access to dictionaries and meaning-related information, but also with improving the users' awareness of the grammatical structures that correspond to their language learning level. As such, the system can be seen as a complementary application to a language course, where the grammatical structures of the user's level will be in focus (by means of text highlighting), with the bonus of having a plethora of new vocabulary available, since it is designed to process any user-chosen, Web-based text.

Some of the grammatical structures that should be highlighted are complex linguistic structures that are not always recognized by parsers and need hand-written rules to cover this lack of parser information. Since these rules are not trivial to implement and require specialized knowledge, in this paper we evaluate the performance of the specialized rules against a gold standard. In specific, we focus on rules precision, because it is a key point of the pedagogical purpose of our system. In addition, we compare our specialized rules performance against parser dependency tagging performance.

This paper is organized as follows: we present information on systems that automatically enhance grammatical structures in Section 2, specially focusing on what type of information and what resources they use for text enhancements; in Section 3, we describe SMILLE, some of its features, and briefly discuss the CEFR and its language learning levels; we then describe the evaluation process of some of SMILLE's features in Section 4; lastly, in Section 5 we present our final remarks.

\section{Related Work}

CALL systems have recently started to use NLP applications for aiding in reading activities (Azab et al., 2013b), so, in this section, we describe a selection of ATICALL systems that use text enhancements for SLA: SmartReader (Azab et al., 2013a,b), WERTi (Meurers et al., 2010), and FLAIR (Chinkina and Meurers, 2016; Chinkina et al., 2016).

The SmartReader provides a reading assistant tool that uses a parser to process texts and highlights information for the user based on the parser analysis. The system presents definitions for content words, grammatical information for function words, and encyclopedic information for named entities. It also displays the syntactic function (such as subject, object) of selected words in the given sentence and generates simple questions about named entities, provided the answers are in the near context.

The WERTi system allows for text enhancements of selected linguistic elements of English, Spanish and German. It also presents exercises corresponding to the selected structure, such as clicking on relevant words, filling the gaps with multiple choice questions or writing the correct word in a gap. The user can choose any URL as input, and WERTi will highlight (by means of color coding) the linguistic structure that was selected by the user or modify the text for testing the user's skills based on the selected activity. It uses parsing combined with rules and regular expressions to retrieve text information for seven linguistic structures.

The FLAIR system is described as an online information retrieval system "that uses efficient algorithms to retrieve, annotate and rerank Web documents based on the grammatical constructions they contain" (Chinkina et al., 2016). FLAIR searches online documents based on keywords selected by the user, parses the first twenty documents retrieved by the search engine and ranks them according to the settings the user selected as most important. It can also recognize 87 different types of grammatical structures described in the official curriculum for German schools. These structures are annotated in the texts and highlighted for the user.

In addition to these systems, there are also other CALL systems that present information from texts to the user, but do not focus on grammatical high- 
lighting. This is the case, for instance, of the REAP system (Brown and Eskenazi, 2004), which is more focused on text retrieval based on user profile and on vocabulary information, and the CoBRA system (Deville et al., 2013), which has a database of aligned multilingual texts and relies on the teacher to select the relevant information for the language learner. There are also systems that were developed within the field of text simplification or readability, but they usually focus on the text properties themselves and on L1 instead of $\mathrm{L} 2$.

\section{System Description}

SMILLE is being developed for English, but there will be an effort to port it to other languages as well. Following the idea of WERTi (Meurers et al., 2010) and the SmartReader (Azab et al., 2013a,b), SMILLE was designed in a way that the users have independence for choosing online reading materials in the foreign language, so that the freedom of choice should serve as an incentive for further developing the learning process. Using the selected, Web-based text, SMILLE provides a reading assistant module that helps the user to notice linguistic content of the target language by highlighting (i.e., enhancing) language structures in context, while also offering the possibility of looking up meaning and word class.

In the way it presents the text enhancements and the features of the reading assistant module, SMILLE bears some similarities with the systems presented in Section 2. However, it distinguishes itself in how the enhancements are selected. For instance, in WERTi, only a few grammatical structures are highlighted for the user, and, in SmartReader, only information more relevant to the meaning of the lexical units is presented, and only parser information is shown as part of the grammatical training. And, while FLAIR and SMILLE share a bigger scope in terms of enhancements, the types and granularity of grammatical structures are different (for instance, FLAIR does not distinguish between gerund and present participle, but SMILLE does). In addition, SMILLE links the displayed information to the guidelines of the CEFR and to other language learning resources, so that the enhancements are not limited to isolated linguistic structures, but covers the needs for the different language levels. As such, users can read texts that are interesting according to their own preference, while keeping an eye on important information in terms of linguistic structures that are relevant for their process of acquiring a second language.

For retrieving the relevant content in the chosen Web page, SMILLE crawls over the HTML structure and extracts its text content. This text content is then parsed for part-of-speech (PoS) and syntactic dependencies with the Stanford parser (Manning et al., 2014). The parsed text content is then analyzed with hand-written rules for creating new tags for each relevant grammatical structure. After this process, a new Web page is constructed, showing the same information extracted from the original one, but with new HTML code and different JavaScript and CSS scripts that allow for real-time modifications of the text.

Some of the grammatical information that is detected by SMILLE requires only that the parser correctly analyzes the word or structure in question. Such is the case, for instance, of adverbs, adjectives and simple verb tenses. Other structures require some rules for retrieving more complex word formations, such as compound verb tenses, phrasal verbs and passive voice, but the information is mostly retrieved from the dependency and PoS tags. Still other structures though, such as WH-questions and question tags, are retrieved based on rules specifically written for them. As such, SMILLE combines the analysis done by the parser with hand-written rules to extract text information that would not be easily identified, and would not be salient, in a raw input.

\subsection{CEFR Levels}

The Common European Framework of Reference (CEFR) for Languages (Council of Europe, 2011) presents a guide in terms of language levels and content. It provides a description for the communication goals of a language learner.

As a general guide, it leaves various gray areas in which the content of the learning process is not so clear. The information at each level also does not cover the different needs for language learners with different native languages. As such, the specific curricula of different language courses do not need to be necessarily the same regarding the six language mastery levels (Alderson, 2007; Little, 2007).SMILLE is being developed in the framework of a partnership between the Univer- 
sité catholique de Louvain and Altissia ${ }^{1}$, so, our information regarding the grammatical structures and CEFR levels are linked to the language course structure of our enterprise partner.

While developing the system, we had to make a decision regarding the granularity of grammatical structures and the escalation of knowledge associated to each language levels. The CEFR was designed for language learning, but Web-based documents normally don't have this instructive approach by design. In a language course, different grammatical structures can be learned in progressive steps, so, for instance, today a language learner may study the modal verb "can" and later, during another session, it is possible to learn the modal verb "must". In an online text, the chances are that different modal verbs will appear at the same time, interwoven in the text. To address this fine-grained differentiation, SMILLE would have to encompass specific rules for each case, sometimes for each word in a grammatical category. This would require more processing and an undesired increase in the number of rules. So, although the system respects the escalation related to different language levels (e.g., different grammatical structures in levels B1 and B2 were separated in specific rules), the progression in the same level was overruled and generalized.

\subsection{System Resources}

SMILLE is responsible for analyzing a text that was chosen by the user and for outputting an enhanced text. For example, if the user is currently studying phrasal verbs, the system can highlight phrasal verbs in the chosen text, so that the user's attention is more easily drawn to the in-context occurrence of this kind of syntactical construct of the English language. The output of SMILLE is a new Web page that can highlight relevant grammatical structures based on the user's language level, but it can also help the user to understand the meaning of different vocabulary that is present on the text.

For the grammatical content of the text, there is a sidebar menu showing all the grammatical information available on the text that corresponds to a given language level. The highlighting is done in real-time, so that the user can change the highlighted structures on the fly. The highlighted text is modified in terms of color coding (font and background colors) and by changing the format

\footnotetext{
${ }^{1}$ Site: http: //altissia.com/
}

to bold. The option for these three modifications are based on the results of Simard (2009), which have shown that the use of three modifications was among the best ways of enhancing a text structure without saturation.

Our system is designed to be able to show grammatical information corresponding to levels A1 to $\mathrm{C} 1$ of the $\mathrm{CEFR}^{2}$. Depending on the chosen level, a number of grammatical structures will be displayed on the sidebar menu. These structures can vary from simple part-of-speech information, such as adjectives, adverbs, nouns, proper nouns, etc. in level A1 to more complex structures such as the use of gerund as object in B1 or the use of passive voice and phrasal verbs in B2. Figure 1 exemplifies structures that can be highlighted by SMILLE's reading assistant module.

If the user wants to look up the meaning of a word, SMILLE offers two possibilities: if it is not a content word, there is the possibility to look it up online on the Merriam-Webster ${ }^{3}$; on the other hand, if it is a content word, then both the Merriam-Webster's definitions and the WordNet's (Fellbaum, 1998) glosses can be looked up online ${ }^{4}$. The access to both online resources was implemented by CTRL-clicking on the desired word and selecting the desired resource from a context menu. In addition, by hovering the mouse over a word, the system displays PoS and lemma information. In Figure 2, the word speculative was selected and the information from two online resources (Merriam-Webster and WordNet) is displayed; lemma speculative and $\mathrm{PoS}$ adjective of the word are also shown in the form of a tooltip.

The CTRL-click menu has yet another function, and it is related to the grammar information. Any word that is part of a grammatical structure that can be highlighted also has a link to a database of grammatical rules. Since the clicked-on word also belongs to a relative clause, by hovering over the relative clause menu, the gerund sub-menu would collapse and a new sub-menu would be displayed with links to information on relative clauses. This provides for further grammatical training when needed by the user.

\footnotetext{
${ }^{2}$ Level $\mathrm{C} 2$ is focused on reviewing the grammatical knowledge acquired in previous levels.

${ }^{3}$ Site: www.merriam-webster.com/

${ }^{4}$ The WordNet was included as a lexical resource because it presents semantic relations between words, such as hypernyms, meronyms, that are usually not present in a regular dictionary.
} 


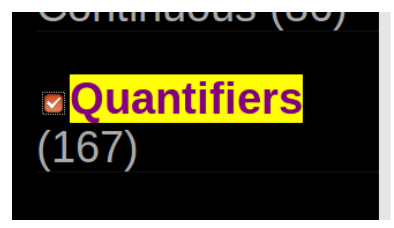

containing multilingual $\mathrm{g}$ just a few years ago, tra minute transmission of th message took less than a

True, some signals ema incidental leakare of radi

Figure 1: Example of highlighting of quantifiers in the text

\section{System Evaluation}

The system was evaluated to verify its performance in retrieving grammatical information with rules. Since SMILLE has language learners as target users, and has a pedagogical function as goal, it is important that the information highlighted on the text really corresponds to the grammatical categories selected by the user. If the system has too low precision, than it may impair the user's capacity of understanding the correct use of the different grammatical structures.

For the evaluation process, a gold standard was manually annotated with the required information. The necessity to create a gold standard is due to the fact that, to our knowledge, there exists no corpus annotated with more complex grammatical structures. There do exist many corpora annotated with PoS and dependency information, but those do not present information such as the ones we are working with here. So, in this section, we first describe our gold standard and the annotation process, and later present and discuss the results of the evaluation.

\subsection{Gold Standard}

The gold standard comprises online newspaper articles and excerpts from literary texts. The newspaper articles were extracted from the sites of The New York Times ${ }^{5}$ (20 articles; 31k tokens), The Guardian $^{6}$ (20 articles; 18k tokens), The Washington Post ${ }^{7}$ (10 articles; 23k tokens), and the USA Today $^{8}$ (5 articles; 9k tokens). These were selected for being dedicated to nation wide and international news, and the articles were collected in a time period that starts at the beginning of March 2017 and ends at the beginning of April 2017. Since newspaper articles generally do not present much dialogue and interaction, and some of the grammatical structures (e.g., short answers,

\footnotetext{
${ }^{5}$ Site: wWw. nytimes. com.

${ }^{6}$ Site: www . theguardian. com.

${ }^{7}$ Site: www. washingtonpost. com.

${ }^{8}$ Site: wWw. usatoday. com.
}

WH-questions, question tags) appear more often in dialogues, we further selected seven literary texts from the Project Gutenberg ${ }^{9}$ which contained longer dialogues: The Yellow Sign (9k tokens), Varney, the Vampyre (chapters II to V; 20k tokens), The Great God Pan (chapter V; 11k tokens), and An Ideal Husband (First Act; 2k tokens).

The corpus was annotated with the following grammatical structures: (1) phrasal verbs, (2) relative clauses, (3) WH-questions, (4) question tags, (5) short answers, (6) gerunds, (7) present participles, (8) infinitives with "to", and (9) infinitives without the particle "to". Most of these structures were selected because they are not retrievable based only on PoS or dependency information, and, although phrasal verbs are indeed recognized based on dependency information, we decided to include this structure for its importance in the learning of English as second language. Each type of grammatical structure was annotated following specific criteria, which were laid out in an annotation manual. Since some grammatical structures are rarer than others, the annotation was not done equally in all texts. Structures that are not abundant in most texts (i.e., wh-questions, question tags, short answers, and phrasal verbs) were annotated in all documents of the corpus, whenever they appeared. The other ones (i.e., gerunds, present participles, infinitives with and without "to", and relative clauses) were annotated in only a part of the corpus. Since there is this different distribution along the corpus, Table 1 presents the number of documents (newspaper articles and literary texts) that contains the structure and the token and sentence count in the gold standard.

Table 1 shows how many structures were annotated in the gold standard. As can be seen, even though the whole corpus was scrutinized in the search for certain structures, we couldn't find many instances of question tags and short answers.

\subsection{Evaluation}

The evaluation was made on a sentence basis. Table 2 shows precision, recall and F-measure for each of the nine tested grammatical structures.

The overall precision, recall and f-measure of the rule-based annotation ${ }^{10}$ was, respectively, $85.20 \%, 82,40 \%$ and $83.78 \%$. Since the system

\footnotetext{
${ }^{9}$ Site: www . gutenberg. org.

${ }^{10}$ This excludes the evaluation of phrasal verbs, which was just based on parser information.
} 


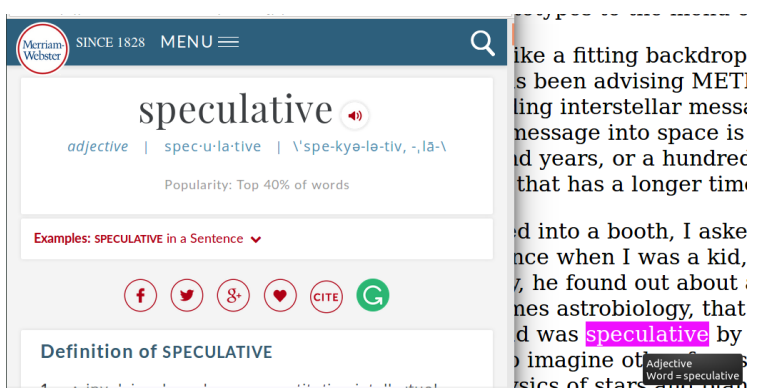

(a) Merriam-Webster's definitions

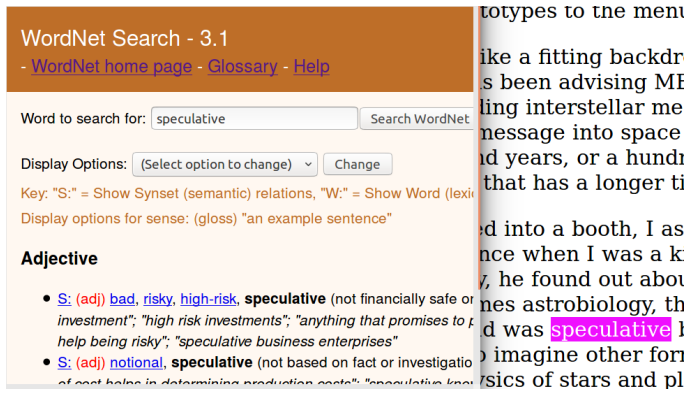

(b) WordNet's glosses

Figure 2: SMILLE can show dictionary information for a given word.

Table 1: Gold standard description

\begin{tabular}{|l|r|r|r|r|}
\hline Grammatical Structure & \# Texts & \# Sentences & \# Tokens & \# Structures Annotated \\
\hline Phrasal Verb & 58 & 6,052 & $121.1 \mathrm{k}$ & 534 \\
\hline Infinitive with "to" & 14 & 1,478 & $31.9 \mathrm{k}$ & 444 \\
\hline Infinitive without "to" & 13 & 1,477 & $31.8 \mathrm{k}$ & 564 \\
\hline Gerund & 26 & 3,920 & $66.8 \mathrm{k}$ & 282 \\
\hline Present Participle & 13 & 2,443 & $35.0 \mathrm{k}$ & 453 \\
\hline Relative Clause & 34 & 2,217 & $56.7 \mathrm{k}$ & 425 \\
\hline WH-Question & 20 & 4,044 & $66.9 \mathrm{k}$ & 176 \\
\hline Question Tag & 3 & 1,992 & $22.6 \mathrm{k}$ & 25 \\
\hline Short Answer & 7 & 3,024 & $42.3 \mathrm{k}$ & 42 \\
\hline
\end{tabular}

Table 2: The SMILLE's Evaluation: Precision, Recall and F-measure

\begin{tabular}{|l|c|c|c|}
\hline Grammatical Structure & $\mathbf{P}$ & $\mathbf{R}$ & $\mathbf{F}$ \\
\hline Phrasal Verbs & .951 & .673 & .878 \\
\hline Gerunds & .879 & .697 & .778 \\
\hline Present Participles & .891 & .799 & .843 \\
\hline Infinitives with "to" & .983 & .859 & .917 \\
\hline Infinitives without "to" & .840 & .979 & .904 \\
\hline Relative Clauses & .870 & .780 & .823 \\
\hline WH-Questions & .957 & .779 & .859 \\
\hline Question Tags & .905 & .760 & .826 \\
\hline Short Answers & .215 & .676 & .327 \\
\hline
\end{tabular}

is focused on the learning process, the higher precision score is positive, because showing correct structures is more important than showing a lot of structures that may be incorrect. So, as Meurers et al. (2010) point out, in CALL, the balance tends to be more inclined to the precision side, and less to recall.

The results showed an f-measure that is above the mean $(\alpha=0.05)$ for generating labeled dependency parsing (Cer et al., 2010). We also compared the performance of our rules for rela- tive clauses with the performance of an annotation solely based on the relative clause annotation that the Stanford parser provides, and the annotation based only on the parser had $59.32 \%$ of precision, $94.04 \%$ of recall and $72.75 \%$ of f-measure, which ranks below the annotation with our rules.

In terms of comparison to state-of-the-art systems, the only other work we know of that presents a similar evaluation is FLAIR (Chinkina and Meurers, 2016), which used 9 newspaper articles as corpus to evaluate 87 different rules used in the FLAIR system for retrieving grammatical information from web-based texts. Unfortunately, the rules and the evaluation process were different than the ones presented here. For instance, Chinkina and Meurers (2016) did not separate gerunds and present participles, considering both as "-ing verb forms"; thus, they use a rule that is more broad in scope than ours and do not have the problem of distinguishing between the structures. So, although some of the patterns are probably similar, such as the ones for $\mathrm{WH}$-questions and question tags, the different corpora that were used represent a hindrance for a more detailed comparison.

To better understand the results we got from the 


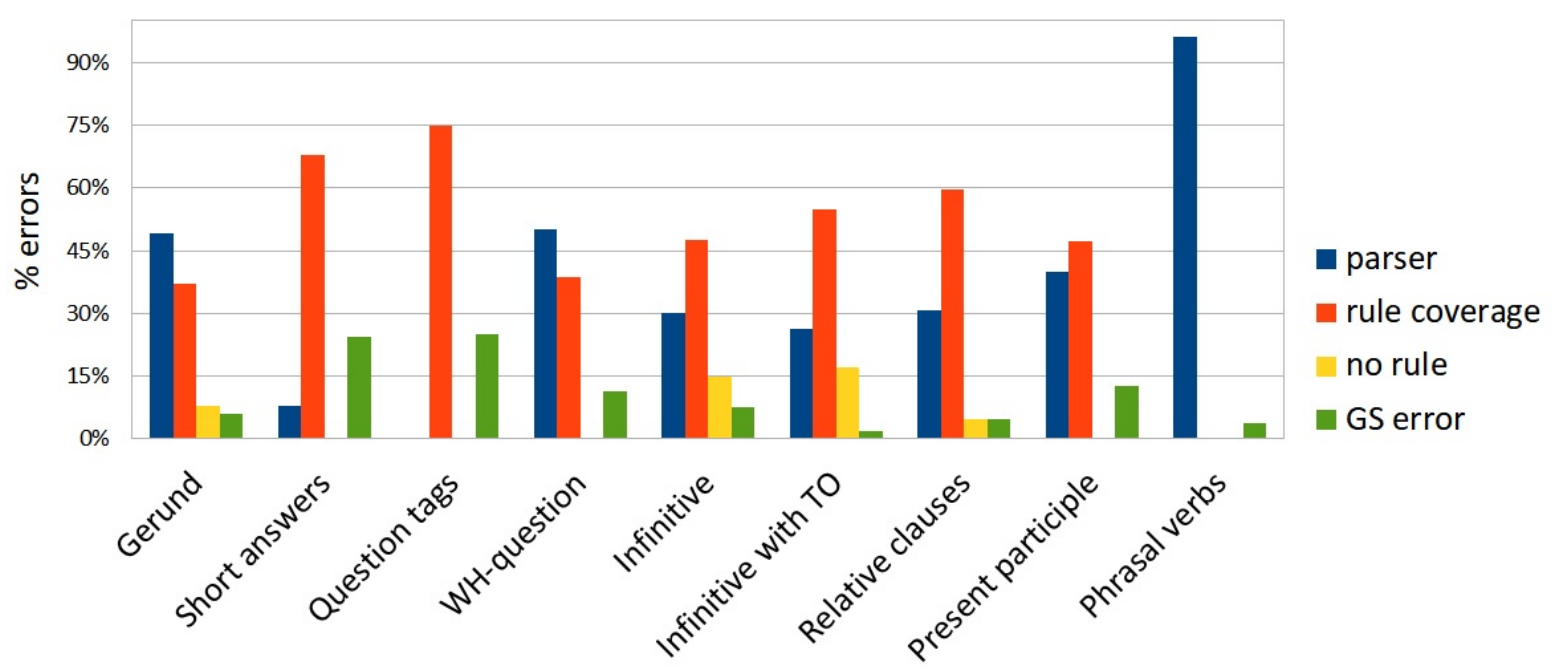

Figure 3: Error analysis

evaluation, we conducted an error analysis. The errors (false positives and false negatives) of the evaluated structures were classified into four categories: parser (parsing error), rule coverage (the rules do not address the specific structure or are too permissive in scope), no rule (there is no rule designed for the structure), GS error (gold standard annotation error), as can be seen in Figure 3 . While errors made by the parser annotation (in which some of the rules are based) are responsible for a great part (48\%) of the errors, the coverage of our rules (responsible for $38 \%$ of the errors) was the main reason for the lack of recall of the system and, especially, for the low precision we got for short answers (in this case, because of a single, too permissive rule). The errors in the gold standard annotation, while not very frequent, prevented, for instance, that our question tag precision reached $100 \%$ (which would be the correct precision for this type of structure). This error analysis was a very important step for the future developments of the system, since it allowed us to better pinpoint where there is room for improvement in the rules.

\section{Conclusion}

This paper presented a system that combines available NLP resources for enhancing language learning. SMILLE extracts raw text from a Web page chosen by the user and enhances its contents by providing the user with tools for a more independent language learning that can take place anywhere with available Internet access. The text is parsed, and a set of rules specific for the different grammatical structures is then applied. By processing texts with NLP tools and rules, the system presents a series of linguistic information, aiding the user to notice in-context grammatical structures and providing access to glosses and definitions for words, while also offering grammatical explanations for specific types of structures.

SMILLE performed above $85 \%$ in terms of precision in most of the evaluated grammatical structures, but lacked a bit in terms of recall. The case of short answers can be considered an outlier, since it presented a very low precision, and so will require special attention. Future improvements will take into account the full error analysis that was carried out and that pinpointed how the flaws in the rules can be addressed.

In terms of the system as a whole, further developments have to be implemented to cover other languages, and to create a database for keeping track of user information and user activity concerning language learning (e.g., words that are looked up in dictionaries, grammatical rules consulted, etc.). With these features in place, SMILLE will be able to provide a more customizable user experience.

\section{Acknowledgments}

The authors would like to thank the Walloon Region (Projects BEWARE n. 1510637 and 1610378) for support, and Altissia International for research collaboration. 


\section{References}

J Charles Alderson. 2007. The cefr and the need for more research. The Modern Language Journal 91(4):659-663.

Mahmoud Azab, Ahmed Salama, Kemal Oflazer, Hideki Shima, Jun Araki, and Teruko Mitamura. 2013a. An english reading tool as a nlp showcase. In The Companion Volume of the Proceedings of IJCNLP 2013: System Demonstrations. Asian Federation of Natural Language Processing, Nagoya, Japan, pages 5-8. http://www.aclweb.org/anthology/I13-2002.

Mahmoud Azab, Ahmed Salama, Kemal Oflazer, Hideki Shima, Jun Araki, and Teruko Mitamura. 2013b. An nlp-based reading tool for aiding nonnative english readers. Recent Advances in Natural Language Processing page 41.

Jonathan Brown and Maxine Eskenazi. 2004. Retrieval of authentic documents for reader-specific lexical practice. In InSTIL/ICALL Symposium 2004.

Daniel M Cer, Marie-Catherine De Marneffe, Daniel Jurafsky, and Christopher D Manning. 2010. Parsing to stanford dependencies: Trade-offs between speed and accuracy. In LREC. Floriana, Malta.

Maria Chinkina, Madeeswaran Kannan, and Detmar Meurers. 2016. Online information retrieval for language learning. ACL 2016 page 7.

Maria Chinkina and Detmar Meurers. 2016. Linguistically aware information retrieval: providing input enrichment for second language learners. In Proceedings of the 11th Workshop on Innovative Use of NLP for Building Educational Applications, San Diego, $C A$.

Council of Europe. 2011. Common European Framework of Reference for Languages: learning, teaching, assessment. Cambridge University Press.

Jeremy Cross. 2002. Noticing'in sla: Is it a valid concept. TESL-EJ 6(3):1-9.

Guy Deville, Laurence Dumortier, Jean-Roch Meurisse, and Marc Miceli. 2013. Ressources lexicales pour laide a lapprentissage des langues. Ressources lexicales: contenu, construction, utilisation, évaluation 30:291-311.

Catherine Doughty. 1991. Second language instruction does make a difference. Studies in second language acquisition 13(04):431-469.

Christiane Fellbaum. 1998. WordNet. Wiley Online Library.

David Little. 2007. The common european framework of reference for languages: Perspectives on the making of supranational language education policy. The Modern Language Journal 91(4):645-655.
Christopher D. Manning, Mihai Surdeanu, John Bauer, Jenny Finkel, Steven J. Bethard, and David McClosky. 2014. The Stanford CoreNLP natural language processing toolkit. In Association for Computational Linguistics (ACL) System Demonstrations. pages 55-60. http://www.aclweb.org/anthology/P/P14/P14-5010.

Detmar Meurers. 2012. Natural language processing and language learning. The Encyclopedia of Applied Linguistics .

Detmar Meurers, Ramon Ziai, Luiz Amaral, Adriane Boyd, Aleksandar Dimitrov, Vanessa Metcalf, and Niels Ott. 2010. Enhancing authentic web pages for language learners. In Proceedings of the NAACL HLT 2010 Fifth Workshop on Innovative Use of NLP for Building Educational Applications. Association for Computational Linguistics, pages 10-18.

Luke Plonsky and Nicole Ziegler. 2016. The call-sla interface: Insights from a second-order synthesis .

Hayo Reinders. 2012. Towards a definition of intake in second language acquisition .

Richard Schmidt. 2012. Attention, awareness, and individual differences in language learning. Perspectives on individual characteristics and foreign language education 6:27.

Richard W Schmidt. 1990. The role of consciousness in second language learning1. Applied linguistics 11(2):129-158.

Daphnée Simard. 2009. Differential effects of textual enhancement formats on intake. System 37(1):124-135.

Michael Sharwood Smith. 1993. Input enhancement in instructed sla. Studies in second language acquisition 15(02):165-179.

Mike Sharwood Smith and John Truscott. 2014. Explaining input enhancement: A mogul perspective. International Review of Applied Linguistics in Language Teaching 52(3):253-281.

John Truscott. 1998. Noticing in second language acquisition: A critical review. Second Language Research 14(2):103-135. 